\title{
Solitons, Cnoidal Waves, Snoidal Waves and Other Solutions to Whitham-Broer-Kaup System
}

\author{
A. H. Bhrawy ${ }^{1,2}$, M. A. Abdelkawy ${ }^{2}$, E. M. Hilal ${ }^{3}$, A. A. Alshaery ${ }^{3}$ and A. Biswas ${ }^{1,4, *}$ \\ ${ }^{1}$ Department of Mathematics, Faculty of Science, King Abdulaziz University, Jeddah, Saudi Arabia \\ 2 Department of Mathematics, Faculty of Science, Beni-Suef University, Beni-Suef, Egypt \\ ${ }^{3}$ Department of Mathematics, Faculty of Science for Girls, King Abdulaziz University, Jeddah, Saudi Arabia \\ ${ }^{4}$ Department of Mathematical Sciences, Delaware State University, Dover, DE 19901-2277, USA
}

Received: 8 Aug. 2013, Revised: 5 Nov. 2013, Accepted: 6 Nov. 2013

Published online: 1 Sep. 2014

\begin{abstract}
Using a computerized symbolic computation technique based on improved Jacobi elliptic function method, we find several solutions for Whitham-Broer-Kaup-Like (WBKL) system. These solutions contain hyperbolic, triangular solutions. When the parameters are taken as special values the solitary wave solutions can be obtained for other systems. The traveling wave solutions are also discussed that obtains solitary wave and singular soliton solution.
\end{abstract}

Keywords: Jacobi elliptic functions method, Nonlinear physical phenomena, Broer-Kaup (BK) system, Whitham-Broer-Kaup-Like system, Whitham-Broer-Kaup system, the classical long-wave system, the variant Boussinesq system and the dispersive long wave system

\section{Introduction}

The study of solitons, cnoidal waves, snoidal waves appear everywhere no matter where we look around. For example, it shows up in plasma physics, mathematical biosciences, nuclear physics, theoretical physics, nonlinear fiber optics, fluid dynamics, mathematical chemistry and several others. Out of these areas, the application of solitons is predominantly prominent in the area of nonlinear fiber optics where loads of data are transmitted over transoceanic and transcontinental distances in just a matter of a few femto seconds or rather atto seconds. Thus, the world of information sciences has immensely advanced because of the profound progress in the theory of solitons. Hence it is important and imperative to dig a little deeper in this area of solitons since research in this field has made gigantic leaps in the technology of information sciences. This paper is therefore going to address the study of solitons, cnoidal waves and snoidal waves for a particular nonlinear evolution equation which is the Whitham-Broer-Kaup equation.
The (1+1) Broer-Kaup (BK) system:

$$
\begin{aligned}
& u_{t}+u u_{x}+v_{x}=0, \\
& v_{t}+u_{x}+(u v)_{x}+u_{x x x}=0,
\end{aligned}
$$

is used to model the bi-directional propagation of long waves in shallow water [1]-[3]. In this paper, we study the Whitham-Broer-Kaup-Like (WBKL) system given in the form:

$$
\begin{aligned}
& u_{t}+u u_{x}+\gamma v_{x}+\beta u_{x x}=0, \\
& v_{t}+(u v)_{x}+\alpha u_{x x x}-\beta v_{x x}=0 .
\end{aligned}
$$

We can find special cases of system (2):

1.If $\gamma=1$, System (2) reduce to Whitham-Broer-Kaup system

$$
\begin{aligned}
& u_{t}+u u_{x}+v_{x}+\beta u_{x x}=0, \\
& v_{t}+(u v)_{x}+\alpha u_{x x x}-\beta v_{x x}=0,
\end{aligned}
$$

which is a completely integrable model describes the dispersive long wave in shallow water, where $u(x, t)$ is the field of horizontal velocity, $v(x, t)$ is the height which deviates from the equilibrium position of liquid, $\alpha$ and $\beta$ are constants that represent different diffusion powers [4]-[7].

\footnotetext{
*Corresponding author e-mail: biswas.anjan@gmail.com
} 
2.If $\gamma=1$ and $\alpha=0$, System (2) reduce to the classical long-wave system [8]-[10]:

$$
\begin{aligned}
& u_{t}+u u_{x}+v_{x}+\beta u_{x x}=0 \\
& v_{t}+(u v)_{x}-\beta v_{x x}=0
\end{aligned}
$$

which describe the shallow water wave with diffusion. 3.If $\gamma=\alpha=1$ and $\beta=0$, System (2) reduce to the variant Boussinesq system [11]-[14]:

$$
\begin{aligned}
& u_{t}+u u_{x}+v_{x}=0, \\
& v_{t}+(u v)_{x}+u_{x x x}=0,
\end{aligned}
$$

which is a model for water waves, where $u(x, t)$ and $v(x, t)$ are the velocity and the total depth, respectively.

4.If $\gamma=1, \alpha=\frac{1}{3}$ and $\beta=0$, System (2) reduce to the dispersive long wave system:

$$
\begin{aligned}
& u_{t}+u u_{x}+v_{x}=0, \\
& v_{t}+(u v)_{x}+\frac{1}{3} u_{x x x}=0,
\end{aligned}
$$

where $v(x, t)$ is the elevation of the water wave, $u(x, t)$ is the surface velocity of water along $x$-direction. Solutions of (6) is very helpful for coastal and civil engineers to apply the nonlinear water wave model in a harbor and coastal design [15]- [17].

5.If $\gamma=1, \alpha=\frac{1}{4} \varepsilon^{2}$ and $\beta=0$, integrable Kaup-Boussinesq shallow water system may be obtained [12]. The integrable nonlinear systems are used extensively as approximate models in hydrodynamics. They describe in a relatively simple way the competition between nonlinear and dispersive effects:

$$
\begin{aligned}
& u_{t}+u u_{x}+v_{x}=0, \\
& v_{t}+(u v)_{x}+\frac{1}{4} \varepsilon^{2} u_{x x x}=0,
\end{aligned}
$$

where $v(x, t)$ denotes the height of the water surface above a horizontal bottom and $u(x, t)$ denotes its velocity averaged over depth, describes motion of shallow water.

The nonlinear wave phenomena can be observed in various scientific fields, such as plasma physics, optical fibers, fluid dynamics, chemical physics, etc. The nonlinear wave phenomena can obtained in solutions of nonlinear evolution equations (NEEs). The exact solutions of these NEEs plays an important role in the study of nonlinear phenomena. In the past decades, many methods were developed for finding exact solutions of NEEs as the inverse scattering method [18]- [19], Hirota's bilinear method [20], homogeneous balance method [21], sine-cosine method [22,23], Extended F-expansion method [24] optical soliton-like [25], tanh function methods [26,27], $\frac{G^{\prime}}{G}$ expansion method [12,28], Jacobi and Weierstrass elliptic function method $[29,30]$.
Although Porubov et al. [31] have obtained some exact periodic solutions to some nonlinear wave equations, they use the Weierstrass elliptic function and involve complicated deducing. A Jacobi elliptic function (JEF) expansion method, which is straightforward and effective, was proposed for constructing periodic wave solutions for some nonlinear evolution equations. The essential idea of this method is similar to the tanh method by replacing the tanh function with some JEFs such as $s n$, $c n$ and $d n$. For example, the Jacobi periodic solution in terms of $s n$ may be obtained by applying the $s n$-function expansion. Many similarly repetitious calculations have to be done to search for the Jacobi doubly periodic wave solutions in terms of $c n$ and $d n$ [32].

The objectives of this work are two fold. First, we seek to extend others works to establish new exact solutions of distinct physical structures for the nonlinear system (2). The improved Jacobi elliptic function expansion (IJEFE) method will be used to achieve the first goal. The second goal is to show that the power of the IJEFE method is its ease of use to determine shock or solitary type of solutions.

In this paper, we extend the IJEFE method with symbolic computation to such special equations for constructing their interesting Jacobi doubly periodic wave solutions. It is shown that soliton solutions and triangular periodic solutions can be established as the limits of Jacobi doubly periodic wave solutions. In addition the algorithm that we use here is also a computerizable method, in which generating an algebraic system. Two key procedures and laborious to do by hand. But they can be implemented on a computer with the help of mathematica. The outputs of solving the algebraic system from a computer comprise a list of constants. In general if any of the parameters is left unspecified.

\section{Improved Jacobi's elliptic function method}

In this section, we introduce a simple description of the IJEF method, for a given partial differential equation

$$
G\left(u, u_{x}, u_{t}, u_{x t}, \ldots\right)=0 .
$$

We like to know whether travelling waves (or stationary waves) are solutions of Eq. (8). The first step is to unite the independent variables $x$ and $t$ into one particular variable through the new variable

$$
\zeta=x+v t, \quad u(x, t)=U(\zeta)
$$

where $c$ is wave speed, and reduce Eq. (8) to an ordinary differential equation(ODE)

$$
G\left(U, U^{\prime}, U^{\prime \prime}, U^{\prime \prime \prime}, \ldots\right)=0 .
$$

Our main goal is to derive exact or at least approximate solutions, if possible, for this ODE. For this purpose, let us simply $U$ as the expansion in the form,

$$
u(x, y, t)=U(\zeta)=\sum_{i=0}^{N} a_{i} \psi^{i}+\sum_{i=1}^{N} a_{-i} \psi^{-i}
$$


Table 1: Spectrum of nonlinear waves.

\begin{tabular}{llll}
\hline$A$ & $B$ & $C$ & $\psi(\zeta)$ \\
\hline 1 & $-1-m^{2}$ & $m^{2}$ & $\operatorname{sn}(\zeta) \quad$ od $(\zeta)=\frac{c n(\zeta)}{d n(\zeta)}$ \\
$1-m^{2}$ & $2 m^{2}-1$ & $-m^{2}$ & $\operatorname{cn}(\zeta)$ \\
$m^{2}-1$ & $2-m^{2}$ & -1 & $\operatorname{dn}(\zeta)$ \\
$m^{2}$ & $-1-m^{2}$ & 1 & $\operatorname{ns}(\zeta) \frac{1}{\operatorname{sn}(\zeta)} \quad$ or \\
& & & $\operatorname{dc}(\zeta)=\frac{d n(\zeta)}{\operatorname{cn}(\zeta)}$ \\
$-m^{2}$ & $2 m^{2}-1$ & $1-m^{2}$ & $\operatorname{nc}(\zeta)=\frac{1}{\operatorname{cn}(\zeta)}$ \\
-1 & $2-m^{2}$ & $m^{2}-1$ & $\operatorname{nd}(\zeta)=\frac{1}{d n(\zeta)}$ \\
1 & $2-m^{2}$ & $1-m^{2}$ & $\operatorname{sc}(\zeta)=\frac{\operatorname{sn}(\zeta)}{\operatorname{cn}(\zeta)}$ \\
1 & $2 m^{2}-1$ & $-m^{2}\left(-1-m^{2}\right)$ & $\operatorname{sd}(\zeta)=\frac{\operatorname{sn}(\zeta)}{\operatorname{dn}(\zeta)}$ \\
$1-m^{2}$ & $2-m^{2}$ & 1 & $\operatorname{cs}(\zeta)=\frac{c n(\zeta)}{\operatorname{sn} \zeta)}$ \\
$-m^{2}\left(1-m^{2}\right)$ & $2 m^{2}-1$ & 1 & $\operatorname{ds}(\zeta)=\frac{d n(\zeta)}{\operatorname{sn}(\zeta)}$ \\
$\frac{1}{4}$ & $\frac{1-2 m^{2}}{2}$ & $\frac{1}{4}$ & $\operatorname{ns}(\zeta)+\operatorname{cs}(\zeta)$ \\
$\frac{1-m^{2}}{4}$ & $\frac{1+m^{2}}{m^{2}}$ & $\frac{1-m^{2}}{2^{2}}$ & $\operatorname{nc}(\zeta)+\operatorname{sc}(\zeta)$ \\
$\frac{1}{4}$ & $\frac{m^{2}-2}{2}$ & $\frac{m^{2}}{4}$ & $\operatorname{ns}(\zeta)+\operatorname{ds}(\zeta)$ \\
$\frac{m^{2}}{4}$ & $\frac{m^{2}-2}{2}$ & $\frac{m^{2}}{4}$ & $\operatorname{sn}(\zeta)+i c s(\zeta)$ \\
\hline
\end{tabular}

where

$$
\psi^{\prime}=\sqrt{A+B \psi^{2}+C \psi^{4}},
$$

the highest degree of $\frac{d^{p} U}{d \zeta^{p}}$ is taken as

$$
O\left(\frac{d^{p} U}{d \zeta^{p}}\right)=N+p, \quad p=1,2,3, \cdots
$$

$O\left(U^{q} \frac{d^{p} U}{d \zeta^{p}}\right)=(q+1) N+p, \quad q=0,1,2, \cdots, p=1,2,3, \cdots$

Where $A, B$ and $C$ are constants, and $N$ in Eq. (9) is a positive integer that can be determined by balancing the nonlinear term(s) and the highest order derivatives. Normally $\mathrm{N}$ is a positive integer, so that an analytic solution in closed form may be obtained. Substituting Eqs. (10)- (11) into Eq. (9) and comparing the coefficients of each power of $\psi(\zeta)$ in both sides, to get an over-determined system of nonlinear algebraic equations with respect to $v, a_{0}, a_{1}, \cdots$. Solving the over-determined system of nonlinear algebraic equations by use of Mathematica. The relations between values of $A, B, C$ and corresponding JEF solution $\psi(\zeta)$ of Eq. (10) are given in Table 1. Substitute the values of $A, B, C$ and the corresponding JEF solution $\psi(\zeta)$ chosen from table 1 into the general form of solution, then an ideal periodic wave solution expressed by JEF can be obtained.

Where $\mathrm{cn}(\zeta)$ and $\mathrm{dn}(\zeta)$ are the Jacobi elliptic cosine function and the JEF of the third kind, respectively. And

$$
c n^{2}(\zeta)=1-s n^{2}(\zeta), \quad d n^{2}(\zeta)=1-m^{2} s n^{2}(\zeta)
$$

with the modulus $\mathrm{m}(0<m<1)$.

When $\mathrm{m} \longrightarrow 1$, the Jacobi functions degenerate to the hyperbolic functions, i.e.,

$$
\operatorname{sn} \zeta \longrightarrow \tanh \zeta, \quad \operatorname{cn} \zeta \longrightarrow \operatorname{sech} \zeta, \quad d n \zeta \longrightarrow \operatorname{sech} \zeta
$$

when $\mathrm{m} \longrightarrow 0$, the Jacobi functions degenerate to the triangular functions, i.e.,

$$
\operatorname{sn} \zeta \longrightarrow \sin \zeta, \quad \operatorname{cn} \zeta \longrightarrow \cos \zeta \quad \text { and } \quad d n \longrightarrow 1 \text {. }
$$

\section{Whitham-Broer-Kaup like system}

We first consider the WBKL system (2)

$$
\begin{aligned}
& u_{t}+u u_{x}+\gamma v_{x}+\beta u_{x x}=0, \\
& v_{t}+(u v)_{x}+\alpha u_{x x x}-\beta v_{x x}=0,
\end{aligned}
$$

if we use $\zeta=x+v t$ carries system (15) into the system of ODEs

$$
\begin{aligned}
& v U^{\prime}+U U^{\prime}+\gamma V^{\prime}+\beta U^{\prime \prime}=0, \\
& V^{\prime}+(U V)^{\prime}+\alpha U^{\prime \prime \prime}-\beta V^{\prime \prime}=0,
\end{aligned}
$$

where by integrating once we obtain, upon setting the constant of integration to zero,

$$
\begin{aligned}
& v U+\frac{U^{2}}{2}+\gamma V+\beta U^{\prime}=0 \\
& V+U V+\alpha U^{\prime \prime}-\beta V^{\prime}=0
\end{aligned}
$$

if we use the first equation in (17) into the second one, we find

$$
\left(2 \alpha \gamma+\beta^{2}\right) U^{\prime \prime}-U^{3}-3 v U^{2}-2 v^{2} U=0 .
$$

Balancing the term $U^{\prime \prime}$ with the term $U^{3}$ we obtain $N=1$ then

$$
U(\zeta)=a_{0}+a_{1} \psi+a_{-1} \psi^{-1}, \quad \psi^{\prime}=\sqrt{A+B \psi^{2}+C \psi^{4}} .
$$

Substituting Eq. (19) into Eq. (18) and comparing the coefficients of each power of $\psi$ in both sides, to get an over-determined system of nonlinear algebraic equations with respect to $v, a_{i}, i=1,-1$. Solving the over-determined system of nonlinear algebraic equations by use of Mathematica, we obtain three groups of constants:

1.

$$
\begin{aligned}
& a_{-1}= \pm \sqrt{2 A\left(\beta^{2}+2 \alpha \gamma\right)}, \quad v= \pm i \sqrt{-B\left(\beta^{2}+2 \alpha \gamma\right)}, \\
& a_{1}=0 \quad \text { and } \quad a_{0}= \pm i \sqrt{B\left(\beta^{2}+2 \alpha \gamma\right)}
\end{aligned}
$$

2.

$$
\begin{aligned}
& a_{1}= \pm \sqrt{2 C\left(\beta^{2}+2 \alpha \gamma\right)}, \quad a_{-1}= \pm \sqrt{2 A\left(\beta^{2}+2 \alpha \gamma\right)} \\
& v=a_{0}= \pm \sqrt{\left(\beta^{2}+2 \alpha \gamma\right)(6 \sqrt{A C}-B)}
\end{aligned}
$$


3.

$$
\begin{aligned}
& a_{1}= \pm \sqrt{2 C\left(\beta^{2}+2 \alpha \gamma\right)}, \quad a_{-1}=\mp \sqrt{2 A\left(\beta^{2}+2 \alpha \gamma\right)}, \\
& v=a_{0}= \pm i \sqrt{\left(\beta^{2}+2 \alpha \gamma\right)(6 \sqrt{A C}+B)}
\end{aligned}
$$

for each group of constant, we find sixteen solutions of (15), for example for group 1 we find:-

$$
\begin{aligned}
u_{1}= & \pm\left(\sqrt{\left(1+m^{2}\right) \gamma_{1}}+\frac{\sqrt{2 \gamma_{1}}}{s n\left(x \pm i \sqrt{\left(1+m^{2}\right) \gamma_{1} t}\right)}\right) \\
v_{1}= & \pm \frac{1}{\gamma}\left[ \pm i \sqrt{\left(1+m^{2}\right) \gamma_{1}} \times\right. \\
& \left(-\sqrt{\left(1+m^{2}\right) \gamma_{1}}+\frac{\sqrt{2 \gamma_{1}}}{\operatorname{sn}\left(x \pm i \sqrt{\left(1+m^{2}\right) \gamma_{1}} t\right)}\right) \\
& \pm \frac{i \sqrt{\left(1+m^{2}\right) \gamma_{1}}}{2} \times \\
& \left(-\sqrt{\left(1+m^{2}\right) \gamma_{1}}+\frac{\sqrt{2 \gamma_{1}}}{\operatorname{sn}\left(x \pm i \sqrt{\left(1+m^{2}\right) \gamma_{1}} t\right)}\right)^{2} \\
& \left.-\frac{\beta \sqrt{2 \gamma_{1}} c n\left(x \pm i \sqrt{\left.\left(1+m^{2}\right) \gamma_{1} t\right) d n\left(x \pm i \sqrt{\left(1+m^{2}\right) \gamma_{1}} t\right)}\right]}{\operatorname{sn}\left(x \pm i \sqrt{\left(1+m^{2}\right) \gamma_{1}} t\right)^{2}}\right]
\end{aligned}
$$

where $\gamma_{1}=\left(\beta^{2}+2 \alpha \gamma\right)$

$$
\begin{aligned}
u_{2} & = \pm\left(\sqrt{\left(1+m^{2}\right) \gamma_{1}}+\frac{\sqrt{2 m^{2} \gamma_{1}}}{n s\left(x \pm i \sqrt{\left(1+m^{2}\right) \gamma_{1}} t\right)},\right. \\
v_{2} & = \pm \frac{1}{\gamma}\left[ \pm i \sqrt{\left(1+m^{2}\right) \gamma_{1}} \times\right. \\
& \left(\frac{-\sqrt{2 m^{2} \gamma_{1}}}{n s\left(x \pm i \sqrt{\left(1+m^{2}\right) \gamma_{1}} t\right)}-\sqrt{\left(1+m^{2}\right) \gamma_{1}}\right) \\
& \pm \frac{i \sqrt{\left(1+m^{2}\right) \gamma_{1}}}{2} \times \\
& \left(\frac{\sqrt{2 m^{2} \gamma_{1}}}{n s\left(x \pm i \sqrt{\left(1+m^{2}\right) \gamma_{1}} t\right)}-\sqrt{\left(1+m^{2}\right) \gamma_{1}}\right)^{2} \\
& \left.-\frac{\beta \sqrt{\left.2 m^{2} \gamma_{1} c s\left(x \pm i \sqrt{\left(1+m^{2}\right) \gamma_{1}} t\right) d s\left(x \pm i \sqrt{\left(1+m^{2}\right) \gamma_{1}} t\right)\right)}}{n s\left(x \pm i \sqrt{\left(1+m^{2}\right) \gamma_{1}} t\right)^{2}}\right]
\end{aligned}
$$

$$
\begin{aligned}
u_{3}= & \pm\left(\sqrt{\left(1+m^{2}\right) \gamma_{1}}+\frac{\sqrt{2 \gamma_{1}}}{c d\left(x \pm i \sqrt{\left(1+m^{2}\right) \gamma_{1} t}\right)},\right. \\
v_{3}= & \pm \frac{1}{\gamma}\left[ \pm i \sqrt{\left(1+m^{2}\right) \gamma_{1}} \times\right. \\
& \left(-\sqrt{\left(1+m^{2}\right) \gamma_{1}}+\frac{\sqrt{2 \gamma_{1}}}{c d\left(x \pm i \sqrt{\left(1+m^{2}\right) \gamma_{1}} t\right)}\right) \\
& \pm \frac{i \sqrt{\left(1+m^{2}\right) \gamma_{1}}}{2} \times \\
& \left(-\sqrt{\left(1+m^{2}\right) \gamma_{1}}+\frac{\sqrt{2 \gamma_{1}}}{c d\left(x \pm i \sqrt{\left(1+m^{2}\right) \gamma_{1} t}\right)}\right)^{2} \\
& +(m-1) \beta \sqrt{2(m-1) \gamma_{1}} \times \\
& \left.\frac{n d\left(x \pm i \sqrt{\left(1+m^{2}\right) \gamma_{1}} t\right) s d\left(x \pm i \sqrt{\left(1+m^{2}\right) \gamma_{1}} t\right)}{c d\left(x \pm i \sqrt{\left(1+m^{2}\right) \gamma_{1}} t\right)^{2}}\right]
\end{aligned}
$$

$$
\begin{aligned}
u_{4}= & \pm\left(\sqrt{\left(1-2 m^{2}\right) \gamma_{1}}+\frac{\sqrt{2\left(1-m^{2}\right) \gamma_{1}}}{d n\left(x \pm \sqrt{\left(2 m^{2}-1\right) \gamma_{1}} t\right)}\right. \\
v_{4}= & \pm \frac{1}{\gamma}\left[\sqrt{\left(2 m^{2}-1\right) \gamma_{1}} \times\right. \\
& \left(-\sqrt{\left(1-2 m^{2}\right) \gamma_{1}}+\frac{\sqrt{2\left(1-m^{2}\right) \gamma_{1}}}{d n\left(x \pm \sqrt{\left(2 m^{2}-1\right) \gamma_{1} t}\right)}\right) \\
& \pm \frac{\sqrt{\left(2 m^{2}-1\right) \gamma_{1}}}{2} \times \\
& \left(-\sqrt{\left(1-2 m^{2}\right) \gamma_{1}}+\frac{\sqrt{2\left(1-m^{2}\right) \gamma_{1}}}{d n\left(x \pm \sqrt{\left(2 m^{2}-1\right) \gamma_{1} t}\right)}\right)^{2} \\
& +m \beta \sqrt{2\left(1-m^{2}\right) \gamma_{1}} \times \\
& \left.\frac{c n\left(x \pm \sqrt{\left(2 m^{2}-1\right) \gamma_{1}} t\right) s n\left(x \pm \sqrt{\left(2 m^{2}-1\right) \gamma_{1}} t\right)}{d n\left(x \pm \sqrt{\left(2 m^{2}-1\right) \gamma_{1}} t\right)^{2}}\right]
\end{aligned}
$$

$$
\begin{aligned}
u_{5}= & \pm\left(\sqrt{\left(-2+m^{2}\right) \gamma_{1}}+\frac{\sqrt{2\left(-1+m^{2}\right) \gamma_{1}}}{d n\left(x \pm \sqrt{\left(2-m^{2}\right) \gamma_{1}} t\right)}\right. \\
v_{5}= & \pm \frac{1}{\gamma}\left[\sqrt{\left(2-m^{2}\right) \gamma_{1}} \times\right. \\
& \left(\sqrt{\left(-2+m^{2}\right) \gamma_{1}}+\frac{\sqrt{2\left(-1+m^{2}\right) \gamma_{1}}}{d n\left(x \pm \sqrt{\left(2-m^{2}\right) \gamma_{1}} t\right)}\right) \\
& \pm \frac{\sqrt{\left(2-m^{2}\right) \gamma_{1}}}{2} \times \\
& \left(\sqrt{\left(-2+m^{2}\right) \gamma_{1}}+\frac{\sqrt{2\left(-1+m^{2}\right) \gamma_{1}}}{d n\left(x \pm \sqrt{\left(2-m^{2}\right) \gamma_{1}} t\right)}\right)^{2} \\
& +m \beta \sqrt{2\left(-1+m^{2}\right) \gamma_{1}} \times \\
& \left.\frac{c n\left(x \pm \sqrt{\left(2-m^{2}\right) \gamma_{1}} t\right) \operatorname{sn}\left(x \pm \sqrt{\left(2-m^{2}\right) \gamma_{1}} t\right)}{d n\left(x \pm \sqrt{\left(2-m^{2}\right) \gamma_{1}} t\right)^{2}}\right]
\end{aligned}
$$




$$
\begin{aligned}
u_{6}= & \pm\left(\sqrt{\left(1+m^{2}\right) \gamma_{1}}+\frac{\sqrt{2 m^{2} \gamma_{1}}}{d c\left(x \pm i \sqrt{\left(1+m^{2}\right) \gamma_{1}} t\right)}\right. \\
v_{6}= & \pm \frac{1}{\gamma}\left[i \sqrt{\left(1+m^{2}\right) \gamma_{1}} \times\right. \\
& \left(\sqrt{\left(1+m^{2}\right) \gamma_{1}}+\frac{\sqrt{2 m^{2} \gamma_{1}}}{d c\left(x \pm i \sqrt{\left(1+m^{2}\right) \gamma_{1}} t\right)}\right) \\
& \left.+\frac{i \sqrt{\left(1+m^{2}\right) \gamma_{1}} \times}{2} \times\right)^{2} \\
& \left(\sqrt{\left(1+m^{2}\right) \gamma_{1}}+\frac{\sqrt{2 m^{2} \gamma_{1}}}{d c\left(x \pm i \sqrt{\left(1+m^{2}\right) \gamma_{1}} t\right)}\right. \\
& +\beta m(1-m) \sqrt{2 \gamma_{1}} \times \\
& \left.\frac{n c\left(x \pm i \sqrt{\left(1+m^{2}\right) \gamma_{1}} t\right) s c\left(x \pm i \sqrt{\left(1+m^{2}\right) \gamma_{1}} t\right)}{d c\left(x \pm i \sqrt{\left(1+m^{2}\right) \gamma_{1}} t\right)^{2}}\right]
\end{aligned}
$$

$$
u_{w 7}= \pm\left(\sqrt{\left(1-2 m^{2}\right) \gamma_{1}}+\frac{\sqrt{2-m^{2} \gamma_{1}}}{n c\left(x \pm \sqrt{\left(2 m^{2}-1\right) \gamma_{1}} t\right)},\right.
$$$$
v_{7}= \pm \frac{1}{\gamma}\left[\sqrt{\left(2 m^{2}-1\right) \gamma_{1}} \times\right.
$$$$
\left(\sqrt{\left(1-2 m^{2}\right) \gamma_{1}}+\frac{\sqrt{2-m^{2} \gamma_{1}}}{n c\left(x \pm \sqrt{\left(2 m^{2}-1\right) \gamma_{1}} t\right)}\right)
$$$$
+\frac{\sqrt{\left(2 m^{2}-1\right) \gamma_{1}}}{2} \times
$$$$
\left(\sqrt{\left(1-2 m^{2}\right) \gamma_{1}}+\frac{\sqrt{2-m^{2} \gamma_{1}}}{n c\left(x \pm \sqrt{\left(2 m^{2}-1\right) \gamma_{1}} t\right)}\right)^{2}
$$$$
-i m \beta \sqrt{2 \gamma_{1}} \times
$$$$
\left.\frac{d c\left(x \pm \sqrt{\left(2 m^{2}-1\right) \gamma_{1}} t\right) s c\left(x \pm \sqrt{\left(2 m^{2}-1\right) \gamma_{1}} t\right)}{n c\left(x \pm \sqrt{\left(2 m^{2}-1\right) \gamma_{1}} t\right)^{2}}\right],
$$

$$
\begin{aligned}
u_{8}= & \pm \sqrt{\left(-2+m^{2}\right) \gamma_{1}}+\frac{\sqrt{2-\beta^{2}-2 \alpha \gamma}}{n d\left(x \pm \sqrt{\left(2-m^{2}\right) \gamma_{1}} t\right)} \\
v_{8}= & \pm \frac{1}{\gamma}\left[\sqrt{\left(2-m^{2}\right) \gamma_{1}} \times\right. \\
& \left(\sqrt{\left(-2+m^{2}\right) \gamma_{1}}+\frac{\sqrt{2-\beta^{2}-2 \alpha \gamma}}{n d\left(x \pm \sqrt{\left(2-m^{2}\right) \gamma_{1}} t\right)}\right) \\
& +\frac{\sqrt{\left(2-m^{2}\right) \gamma_{1}}}{2} \times \\
& \left(\sqrt{\left(-2+m^{2}\right) \gamma_{1}}+\frac{\sqrt{2-\beta^{2}-2 \alpha \gamma}}{n d\left(x \pm \sqrt{\left(2-m^{2}\right) \gamma_{1}} t\right)}\right)^{2} \\
& -i m \beta \sqrt{2 \gamma_{1}} \times \\
& \left.\frac{c d\left(x \pm \sqrt{\left(2-m^{2}\right) \gamma_{1}} t\right) s d\left(x \pm \sqrt{\left(2-m^{2}\right) \gamma_{1}} t\right)}{n d\left(x \pm \sqrt{\left(2-m^{2}\right) \gamma_{1}} t\right)^{2}}\right]
\end{aligned}
$$

$$
\begin{aligned}
u_{9}= & \pm\left(\sqrt{\left(-2+m^{2}\right) \gamma_{1}}+\frac{\sqrt{2 \beta^{2}+2 \alpha \gamma}}{s c\left(x \pm \sqrt{\left(2-m^{2}\right) \gamma_{1}} t\right)},\right. \\
v_{9}= & \frac{1}{\gamma}\left[\sqrt{\left(2-m^{2}\right) \gamma_{1}}\left(\sqrt{\left(-2+m^{2}\right) \gamma_{1}}+\frac{\sqrt{2 \beta^{2}+2 \alpha \gamma}}{s c\left(x \pm \sqrt{\left(2-m^{2}\right) \gamma_{1}} t\right)}\right)\right. \\
& +\frac{\sqrt{\left(2-m^{2}\right) \gamma_{1}}}{2}\left(\sqrt{\left(-2+m^{2}\right) \gamma_{1}}+\frac{\sqrt{2 \beta^{2}+2 \alpha \gamma}}{s c\left(x \pm \sqrt{\left(2-m^{2}\right) \gamma_{1}} t\right)}\right)^{2} \\
& \left.-\frac{\beta \sqrt{2 \gamma_{1}} d c\left(x \pm \sqrt{\left(2-m^{2}\right) \gamma_{1}} t\right) n c\left(x \pm \sqrt{\left(2-m^{2}\right) \gamma_{1}} t\right)}{s c\left(x \pm \sqrt{\left(2-m^{2}\right) \gamma_{1}} t\right)^{2}}\right]
\end{aligned}
$$

$$
u_{10}= \pm\left(\sqrt{\left(1-2 m^{2}\right) \gamma_{1}}+\frac{\sqrt{2 \beta^{2}+2 \alpha \gamma}}{s d\left(x \pm \sqrt{\left(2 m^{2}-1\right) \gamma_{1}} t\right)},\right.
$$$$
v_{10}=\frac{1}{\gamma}\left[\sqrt{\left(2 m^{2}-1\right) \gamma_{1}} \times\right.
$$

$$
\begin{aligned}
& \left(\sqrt{\left(1-2 m^{2}\right) \gamma_{1}}+\frac{\sqrt{2 \beta^{2}+2 \alpha \gamma}}{s d\left(x \pm \sqrt{\left(2 m^{2}-1\right) \gamma_{1}} t\right)}\right) \\
& +\frac{\sqrt{\left(2 m^{2}-1\right) \gamma_{1}}}{2} \times \\
& \left(\sqrt{\left(1-2 m^{2}\right) \gamma_{1}}+\frac{\sqrt{2 \beta^{2}+2 \alpha \gamma}}{s d\left(x \pm \sqrt{\left(2 m^{2}-1\right) \gamma_{1}} t\right)}\right)^{2} \\
& -\beta \sqrt{2 \gamma_{1}} \times \\
& \left.\frac{c d\left(x \pm \sqrt{\left(2 m^{2}-1\right) \gamma_{1}} t\right) n d\left(x \pm \sqrt{\left(2 m^{2}-1\right) \gamma_{1}} t\right)}{s d\left(x \pm \sqrt{\left(2 m^{2}-1\right) \gamma_{1}} t\right)^{2}}\right]
\end{aligned}
$$

$$
\begin{aligned}
u_{11}= & \pm\left(\sqrt{\left(-2+m^{2}\right) \gamma_{1}}+\frac{\sqrt{2\left(1-m^{2}\right) \gamma_{1}}}{c s\left(x \pm \sqrt{\left(2-m^{2}\right) \gamma_{1}} t\right)}\right. \\
v_{11}= & \frac{1}{\gamma}\left[\sqrt{\left(2-m^{2}\right) \gamma_{1}} \times\right. \\
& \left(\sqrt{\left(-2+m^{2}\right) \gamma_{1}}+\frac{\sqrt{2\left(1-m^{2}\right) \gamma_{1}}}{c s\left(x \pm \sqrt{\left(2-m^{2}\right) \gamma_{1} t}\right)}\right) \\
& \left.+\frac{\sqrt{\left(2-m^{2}\right) \gamma_{1}} \times}{2} \times\right)^{2} \\
& \left(\sqrt{\left(-2+m^{2}\right) \gamma_{1}}+\frac{\sqrt{2\left(1-m^{2}\right) \gamma_{1}}}{c s\left(x \pm \sqrt{\left(2-m^{2}\right) \gamma_{1} t}\right)}\right. \\
& +\beta \sqrt{2\left(1-m^{2}\right) \gamma_{1}} \times \\
& \left.\frac{d s\left(x \pm \sqrt{\left(2-m^{2}\right) \gamma_{1}} t\right) n s\left(x \pm \sqrt{\left(2-m^{2}\right) \gamma_{1}} t\right)}{c s\left(x \pm \sqrt{\left(2-m^{2}\right) \gamma_{1}} t\right)^{2}}\right]
\end{aligned}
$$




$$
\begin{aligned}
u_{12}= & \pm\left(\sqrt{\left(1-2 m^{2}\right) \gamma_{1}}+\frac{\sqrt{2-m^{2}\left(1-m^{2}\right) \gamma_{1}}}{d s\left(x \pm \sqrt{\left(2 m^{2}-1\right) \gamma_{1}} t\right)}\right. \\
v_{12}= & \frac{1}{\gamma}\left[\sqrt{\left(2 m^{2}-1\right) \gamma_{1}} \times\right. \\
& \left(\sqrt{\left(1-2 m^{2}\right) \gamma_{1}}+\frac{\sqrt{2-m^{2}\left(1-m^{2}\right) \gamma_{1}}}{d s\left(x \pm \sqrt{\left(2 m^{2}-1\right) \gamma_{1}} t\right)}\right) \\
& +\frac{\sqrt{\left(2 m^{2}-1\right) \gamma_{1}}}{2} \times \\
& \left(\sqrt{\left(1-2 m^{2}\right) \gamma_{1}}+\frac{\sqrt{2-m^{2}\left(1-m^{2}\right) \gamma_{1}}}{d s\left(x \pm \sqrt{\left(2 m^{2}-1\right) \gamma_{1}} t\right)}\right)^{2} \\
& +\beta m \sqrt{2\left(m^{2}-1\right) \gamma_{1}} \times \\
& \left.\frac{c s\left(x \pm \sqrt{\left(2 m^{2}-1\right) \gamma_{1}} t\right) n s\left(x \pm \sqrt{\left(2 m^{2}-1\right) \gamma_{1}} t\right)}{d s\left(x \pm \sqrt{\left(2 m^{2}-1\right) \gamma_{1}} t\right)^{2}}\right]
\end{aligned}
$$

$$
\begin{aligned}
u_{13}= & \pm\left(\sqrt{\left(-0.5+m^{2}\right) \gamma_{1}}+\frac{0.707107 \sqrt{\beta^{2}+2 \alpha \gamma}}{c s(x+v t)+n s(x+v t)},\right. \\
v_{13}= & \frac{1}{\gamma}\left[0.707107 \beta \sqrt{\beta^{2}+2 \alpha \gamma} \times\right. \\
& \frac{(-c s(x+v t) d s(x+v t)-d s(x+v t) n s(x+v t))}{(c s(x+v t)+n s(x+v t))^{2}} \\
& +v\left(\sqrt{\left(-0.5+m^{2}\right) \gamma_{1}}+\frac{0.707107 \sqrt{\beta^{2}+2 \alpha \gamma}}{c s(x+v t)+n s(x+v t)}\right) \\
& \left.+\frac{v}{2}\left(\sqrt{\left(-0.5+m^{2}\right) \gamma_{1}}+\frac{0.707107 \sqrt{\beta^{2}+2 \alpha \gamma}}{c s(x+v t)+n s(x+v t)}\right)^{2}\right], \\
v= & \pm \sqrt{\left(0.5-m^{2}\right) \gamma_{1}},
\end{aligned}
$$

$$
\begin{aligned}
u_{14}= & \pm\left(\sqrt{\left(-0.5-0.5 m^{2}\right) \gamma_{1}}+\frac{\sqrt{2\left(0.25-0.25 m^{2}\right) \gamma_{1}}}{n c(x+v t)+s c(x+v t)}\right. \\
v_{14}= & \pm \frac{1}{\gamma}\left[\left(\beta \sqrt{2\left(0.25-0.25 m^{2}\right) \gamma_{1}} \times\right.\right. \\
& \frac{d c(x+v t) n c(x+v t)+d c(x+v t) s c(x+v t)}{(n c(x+v t)+s c(x+v t))^{2}} \\
& +v\left(\sqrt{\left(-0.5-0.5 m^{2}\right) \gamma_{1}}+\frac{\sqrt{2\left(0.25-0.25 m^{2}\right) \gamma_{1}}}{(n c(x+v t)+s c(x+v t))}\right) \\
& \left.+\frac{v}{2}\left(\frac{\sqrt{2\left(0.25-0.25 m^{2}\right) \gamma_{1}}}{n c(x+v t)+s c(x+v t)}-\sqrt{\left(-0.5-0.5 m^{2}\right) \gamma_{1}}\right)^{2}\right] \\
v= & \pm \sqrt{\left(0.5+0.5 m^{2}\right) \gamma_{1}}
\end{aligned}
$$

$$
\begin{aligned}
& u_{15}= \pm\left(\sqrt{\left(1-0.5 m^{2}\right) \gamma_{1}}+\frac{0.707107 \sqrt{\beta^{2}+2 \alpha \gamma}}{d s(x+v t)+n s(x+v t)},\right. \\
& v_{15}= \pm \frac{1}{\gamma}\left[\left(-0.707107 \beta \sqrt{\beta^{2}+2 \alpha \gamma} \times\right.\right. \\
& \left.\frac{c s(x+v t) d s(x+v t)+c s(x+v t) n s(x+v t)}{(d s(x+v t)+n s(x+v t))^{2}}\right) \\
& +v\left(\sqrt{\left(1-0.5 m^{2}\right) \gamma_{1}}+\frac{0.707107 \sqrt{\beta^{2}+2 \alpha \gamma}}{d s(x+v t)+n s(x+v t)}\right) \\
& \left.+\frac{v}{2}\left(\sqrt{\left(1-0.5 m^{2}\right) \gamma_{1}}+\frac{0.707107 \sqrt{\beta^{2}+2 \alpha \gamma}}{d s(x+v t)+n s(x+v t)}\right)^{2}\right] \text {, } \\
& v=\sqrt{\left(0.5 m^{2}-1\right) \gamma_{1}} \\
& u_{16}= \pm\left(\sqrt{\left(1-0.5 m^{2}\right) \gamma_{1}}+\frac{0.707107 \sqrt{m^{2} \gamma_{1}}}{i c s(x+v t)+\operatorname{sn}(x+v t)},\right. \\
& v_{16}= \pm \frac{1}{\gamma}\left[\left(0.707107 \beta \sqrt{m^{2} \gamma_{1}} \times\right.\right. \\
& \left.\frac{c n(x+v t) d n(x+v t)-i d s(x+v t) n s(x+v t)}{(i c s(x+v t)+\operatorname{sn}(x)+v t)^{2}}\right) \\
& +v\left(\sqrt{\left(1-0.5 m^{2}\right) \gamma_{1}}+\frac{0.707107 \sqrt{m^{2} \gamma_{1}}}{i c s(x+v t)+\operatorname{sn}(x+v t)}\right) \\
& \left.+\frac{v}{2}\left(\sqrt{\left(1-0.5 m^{2}\right) \gamma_{1}}+\frac{0.707107 \sqrt{m^{2} \gamma_{1}}}{i c s(x+v t)+\operatorname{sn}(x+v t)}\right)^{2}\right] \text {, } \\
& v= \pm \sqrt{\left(0.5 m^{2}-1\right) \gamma_{1}}
\end{aligned}
$$

\subsection{Triangular periodic solutions}

Some trigonometric function solutions can be obtained, if the modulus $m$ approaches to zero in Eqs. (23)-(52)

$$
\begin{aligned}
u_{17}= & -\sqrt{\gamma_{1}}+\sqrt{2} \sqrt{\gamma_{1}} \csc (x+v t), \\
v_{17}= & -\frac{1}{\gamma}\left[\sqrt{2} \beta \sqrt{\gamma_{1}} \cot (x+v t) \csc (x+v t)\right. \\
& -v\left(-\sqrt{\gamma_{1}}+\sqrt{2} \sqrt{\gamma_{1}} \csc (x+v t)\right) \\
& \left.-\frac{1}{2} v\left(-\sqrt{\gamma_{1}}+\sqrt{2} \sqrt{\gamma_{1}} \csc (x+v t)\right)^{2}\right], \\
u_{18}= & -\sqrt{\gamma_{1}}+\sqrt{2} \sqrt{\gamma_{1}} \sec (x+v t), \\
v_{18}= & -\frac{1}{\gamma}\left[-v\left(-\sqrt{\gamma_{1}}+\sqrt{2} \sqrt{\gamma_{1}} \sec (x+v t)\right)\right. \\
& -\frac{1}{2} v\left(-\sqrt{\gamma_{1}}+\sqrt{2} \sqrt{\gamma_{1}} \sec (x+v t)\right)^{2} \\
& \left.-\sqrt{2} \beta \sqrt{\gamma_{1}} \sec (x+v t) \tan (x+v t)\right],
\end{aligned}
$$




$$
\begin{aligned}
u_{19}= & -\sqrt{2} i \sqrt{\gamma_{1}}+\sqrt{2} \sqrt{\gamma_{1}} \cot (x+v t), \\
v_{19}= & \frac{1}{\gamma}\left(v\left(-\sqrt{2} i \sqrt{\gamma_{1}}+\sqrt{2} \sqrt{\gamma_{1}} \cot (x+v t)\right)\right. \\
& +\frac{1}{2} v\left(-\sqrt{2} i \sqrt{\gamma_{1}}+\sqrt{2} \sqrt{\gamma_{1}} \cot (x+v t)\right)^{2} \\
& \left.+\sqrt{2} \beta \sqrt{\gamma_{1}} \csc (x+v t)^{2}\right), \\
u_{20}= & -\sqrt{2} i \sqrt{\gamma_{1}}+\sqrt{2} \sqrt{\gamma_{1}} \tan (x+v t), \\
v_{20}= & -\frac{1}{\gamma}\left[-\sqrt{2} \beta \sqrt{\gamma_{1}} \sec (x+v t)^{2}\right. \\
& -v\left(-\sqrt{2} i \sqrt{\gamma_{1}}+\sqrt{2} \sqrt{\gamma_{1}} \tan (x+v t)\right) \\
& \left.-\frac{1}{2} v\left(-\sqrt{2} i \sqrt{\gamma_{1}}+\sqrt{2} \sqrt{\gamma_{1}} \tan (x+v t)\right)^{2}\right],
\end{aligned}
$$

$$
u_{21}=0.707107 \sqrt{\gamma_{1}}((\sin (x+v t)+\tan (x+v t))-i) \text {, }
$$

$$
\begin{aligned}
v_{21}= & -\frac{1}{\gamma}\left(-0.707107 \beta \sqrt{\gamma_{1}}\left(\cos (x+v t)+\sec (x+v t)^{2}\right)\right. \\
& -v\left(0.707107 \sqrt{\gamma_{1}}((\sin (x+v t)+\tan (x+v t))-i)\right) \\
& -\frac{1}{2} v\left(0.707107 \sqrt{\gamma_{1}}((\sin (x+v t)\right. \\
& \left.+\tan (x+v t))-i))^{2}\right),
\end{aligned}
$$

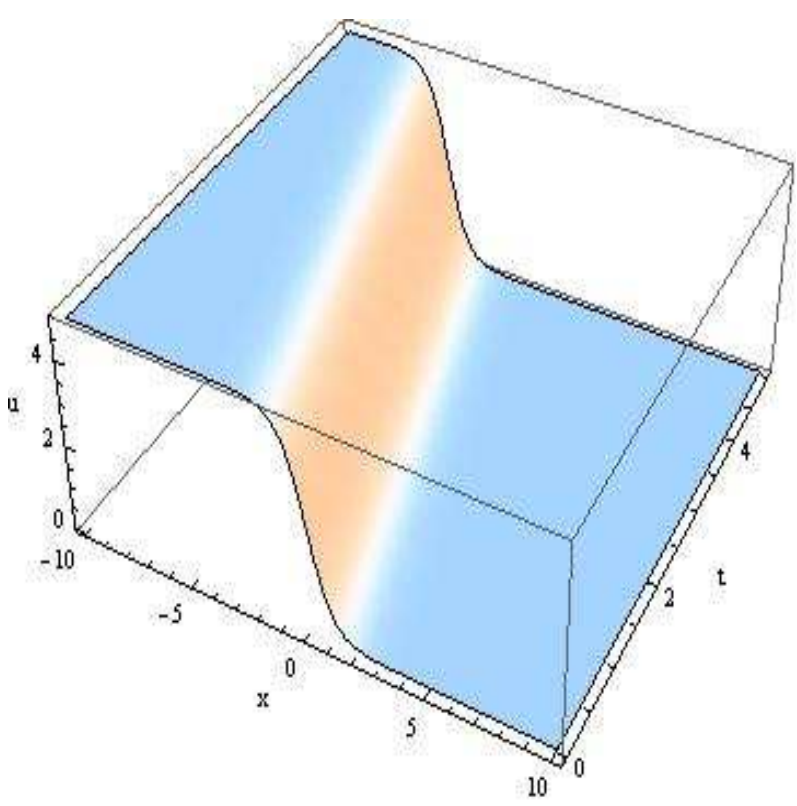

Fig. 1: Three-dimensional of the modulus of solitary wave solution $u_{25}$ (Eq. 47) at $\alpha=\beta=v=\gamma=1$.

$$
\begin{aligned}
u_{22}= & 0.707107 \sqrt{\gamma_{1}}((\cos (x+v t)+\cot (x+v t))-i), \\
v_{22}= & -\frac{1}{\gamma}\left(v\left(0.707107 \sqrt{\gamma_{1}}((\cos (x+v t)+\cot (x+v t))-i)\right)\right. \\
& +\frac{1}{2} v\left(0.707107 \sqrt{\gamma_{1}}((\cos (x+v t)+\cot (x+v t))-i)\right)^{2} \\
& \left.+\beta\left(0.707107 \sqrt{\gamma_{1}}\left(-\csc (x+v t)^{2}-\sin (x+v t)\right)\right)\right),
\end{aligned}
$$

$$
\begin{aligned}
u_{23}= & -\sqrt{\gamma_{1}}+1.41421 \sqrt{\gamma_{1}} \sin (x+v t), \\
v_{23}= & \frac{1}{\gamma}\left(\beta\left(1.41421 \sqrt{\gamma_{1}} \cos (x+v t)\right)\right. \\
& -v\left(\sqrt{\gamma_{1}}-1.41421 \sqrt{\gamma_{1}} \sin (x+v t)\right) \\
& \left.+\frac{1}{2} v\left(\sqrt{\gamma_{1}}-1.41421 \sqrt{\gamma_{1}} \sin (x+v t)\right)^{2}\right) .
\end{aligned}
$$

\subsection{Soliton solutions}

Some solitary wave solutions can be obtained, if the modulus $m$ approaches to 1 in Eqs. (23)-(52)

$$
\begin{aligned}
u_{24}= & -\sqrt{2} \sqrt{\gamma_{1}}+\sqrt{2} \sqrt{\gamma_{1}} \operatorname{coth}(x+v t), \\
v_{24}= & -\frac{1}{\gamma}\left(v\left(\sqrt{2} \sqrt{\gamma_{1}}-\sqrt{2} \sqrt{\gamma_{1}} \operatorname{coth}(x+v t)\right)\right. \\
& -\frac{1}{2} v\left(\sqrt{2} \sqrt{\gamma_{1}}-\sqrt{2} \sqrt{\gamma_{1}} \operatorname{coth}(x+v t)\right)^{2} \\
& \left.+\sqrt{2} \beta \sqrt{\gamma_{1}} \operatorname{csch}(x+v t)^{2}\right),
\end{aligned}
$$

$$
\begin{aligned}
u_{27} & =-i \sqrt{\gamma_{1}}+\sqrt{2} \sqrt{\gamma_{1}} \operatorname{csch}(x+v t), \\
v_{27} & =-\frac{1}{\gamma}\left(\sqrt{2} \beta \sqrt{\gamma_{1}} \operatorname{coth}(x+v t) \operatorname{csch}(x+v t)\right. \\
& -v\left(-i \sqrt{\gamma_{1}}+\sqrt{2} \sqrt{\gamma_{1}} \operatorname{csch}(x+v t)\right) \\
& \left.-\frac{1}{2} v\left(-i \sqrt{\gamma_{1}}+\sqrt{2} \sqrt{\gamma_{1}} \operatorname{csch}(x+v t)\right)^{2}\right),
\end{aligned}
$$




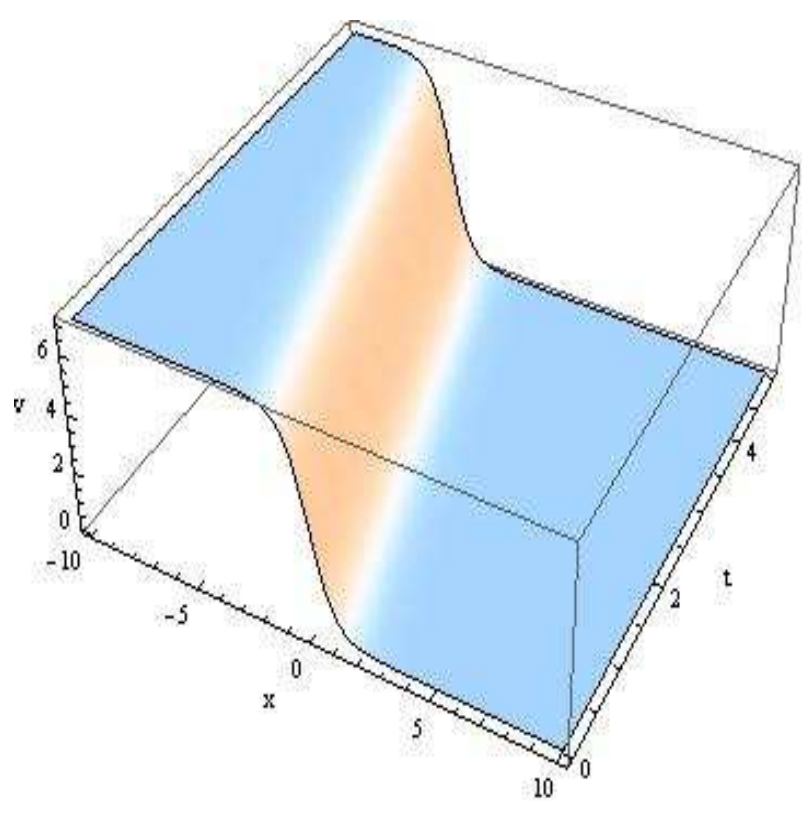

Fig. 2: Three-dimensional of the modulus of solitary wave solution $v_{25}$ (Eq. 47) at $\alpha=\beta=v=\gamma=1$.

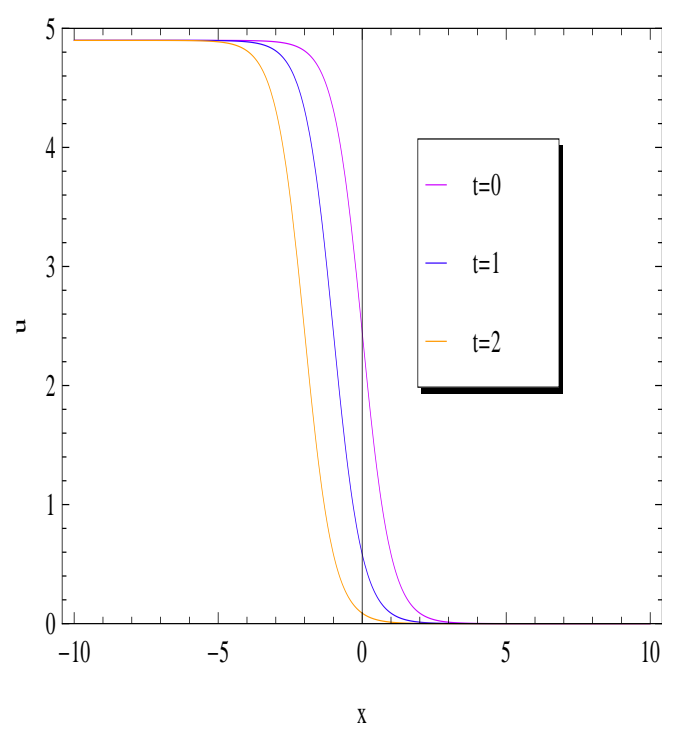

Fig. 3: Graphical representation of of the modulus of solitary wave solution $u_{25}$ (Eq. 47) for $t=0,1$ and 2 at $\alpha=\beta=v=\gamma=$ 1.

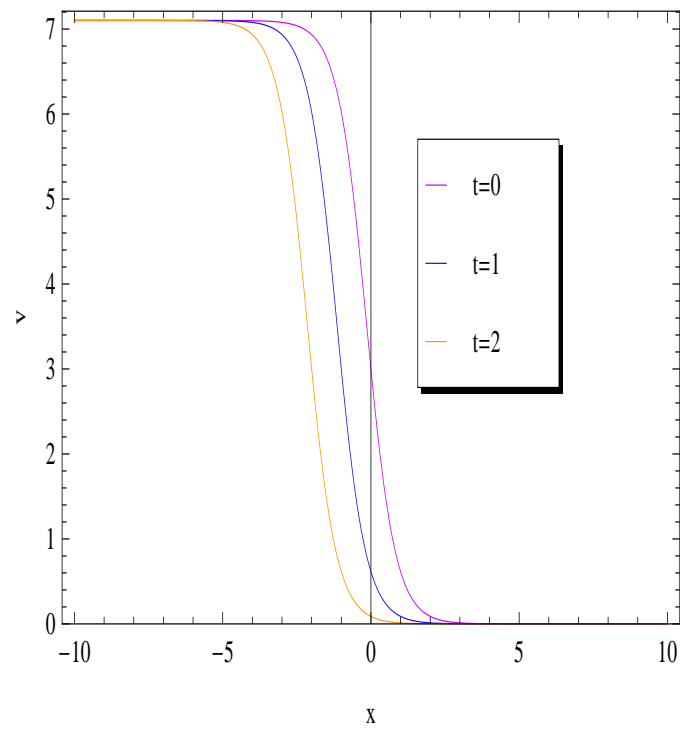

Fig. 4: Graphical representation of of the modulus of solitary wave solution $v_{25}$ (Eq. 47) for $t=0,1$ and 2 at $\alpha=\beta=v=\gamma=$ 1.

$$
\begin{aligned}
u_{28} & =0.707107 \sqrt{\gamma_{1}}(\sinh (x+v t)+\tanh (x+v t)-1), \\
v_{28} & =\frac{0.707107 \sqrt{\gamma_{1}} v}{\gamma}\left[\frac{\beta}{v}\left(\cosh (x+v t)+\operatorname{sech}(x+v t)^{2}\right)\right. \\
& -(\sinh (x+v t)+\tanh (x+v t))-1) \\
& \left.\left.-\frac{0.707107 \sqrt{\gamma_{1}}}{2}(\sinh (x+v t)+\tanh (x+v t)-1)\right)^{2}\right],
\end{aligned}
$$

$$
\begin{aligned}
u_{29} & =0.707107 \sqrt{\gamma_{1}}(\sinh (x+v t)+\tanh (x+v t)-1), \\
v_{29} & =\frac{0.707107 \sqrt{\gamma_{1}} v}{\gamma}\left[\frac{\beta}{v}\left(\cosh (x+v t)+\operatorname{sech}(x+v t)^{2}\right)\right. \\
& +(\sinh (x+v t)+\tanh (x+v t)-1) \\
& \left.+\frac{0.707107 \sqrt{\gamma_{1}}}{2}(\sinh (x+v t)+\tanh (x+v t)-1)^{2}\right],
\end{aligned}
$$

$$
\begin{aligned}
u_{30} & =0.707107 \sqrt{\gamma_{1}}(\operatorname{coth}(x+v t)+i \sinh (x+v t)-1), \\
v_{30} & =\frac{0.707107 \sqrt{\gamma_{1}} v}{\gamma}\left[\frac{\beta}{v}\left(i \cosh (x+v t)-\operatorname{csch}(x+v t)^{2}\right)\right. \\
& +(\operatorname{coth}(x+v t)+i \sinh (x+v t)-1) \\
& \left.+\frac{0.707107 \sqrt{\gamma_{1}}}{2}(\operatorname{coth}(x+v t)+i \sinh (x+v t)-1)^{2}\right] .
\end{aligned}
$$

For above last two groups of constants $(2,3)$ we find another thirty solutions. Three-dimensional of the 
modulus of solitary wave solutions $u_{25}$ and $v_{25}$, are displayed in Figures 1 and 2, respectively, with values of parameters listed in their captions. Moreover, graphical representation for the same solutions are plotted in figures 3 and 4, respectively, with values of parameters listed in their captions.

\section{TRAVELING WAVE SOLUTIONS}

This section will focus on obtaining the traveling wave solutions to (15). The solitary waves as well as the singular soliton solution will be derived by the aid of traveling waves [33]. First of all, (15) is now rewritten in the following pair of coupled equations, after removing the dissipative terms:

$q_{t}+a_{1} q q_{x}+a_{2} r_{x}=0$,

$r_{t}+b_{1}(q r)_{x}+b_{2} q_{x x x}=0$.

The starting point is the traveling wave hypotheses that are

$q(x, t)=g(x-v t)$,

and

$r(x, t)=h(x-v t)$,

where $g$ and $h$ are the wave profiles and $v$ represents the velocity of the traveling waves. Introducing the notation

$s=x-v t$,

equations (53) and (54) transforms to the ordinary differential equations

$2 v g-a_{1} g^{2}-2 a_{2} h=0$,

and

$v h-b_{1} g h-b_{2} g^{\prime \prime}=0$,

respectively, after choosing the integration constant to be zero, since the focus in on obtaining the soliton solution. Here in (58) and (59), the notations $g^{\prime}=d g / d s$ and $g^{\prime \prime}=$ $d^{2} g / d s^{2}$ are utilized. From (58), it is possible to obtain

$h=\frac{1}{2 a_{2}}\left(2 v g-a_{1} g^{2}\right)$.

Substituting (60) into (59) and integrating once, leads to

$\left(g^{\prime}\right)^{2}=\frac{v^{2}}{a_{2} b_{2}} g^{2}-\frac{\left(a_{1}+2 b_{1}\right) v}{3 a_{2} b_{2}} g^{3}+\frac{a_{1} b_{1}}{4 a_{2} b_{2}} g^{4}$,

after simplification, where the integration constant is, once again, taken to be zero. Now from (61), separating variables and integrating one more time, leads to the 1-soliton solution

$g(x-v t)=\frac{A}{D+\cosh [B(x-v t)]}$,

where the amplitude $A$ of the soliton is given by

$A=\frac{6 v}{\sqrt{\left(a_{1}+2 b_{1}\right)^{2}-9 a_{1} b_{1}}}$, and the inverse width $B$ is

$B=\frac{v}{\sqrt{a_{2} b_{2}}}$,

while the parameter $D$ is given by

$D=\frac{a_{1}+2 b_{1}}{\sqrt{\left(a_{1}+2 b_{1}\right)^{2}-9 a_{1} b_{1}}}$.

The definitions of the parameter given by (63)-(65) leads to the constraint conditions

$a_{2} b_{2}>0$,

and

$\left(a_{1}+2 b_{1}\right)^{2}>9 a_{1} b_{1}$.

Finally, the wave profile for $h(x-v t)$ can be obtained from (60).

Integration of (61) also leads to the singular 1-soliton solution that is given by

$g(x-v t)=\frac{A}{D-\sinh [B(x-v t)]}$.

In this case, the free parameters $A$ and $D$ are respectively given by

$A=\frac{6 v}{\sqrt{9 a_{1} b_{1}-\left(a_{1}+2 b_{1}\right)^{2}}}$.

and

$D=\frac{a_{1}+2 b_{1}}{\sqrt{9 a_{1} b_{1}-\left(a_{1}+2 b_{1}\right)^{2}}}$.

while the free parameter $B$, in this case stays the same as (64). These free parameters from (69) and (70) therefore introduce the constraint

$\left(a_{1}+2 b_{1}\right)^{2}<9 a_{1} b_{1}$,

which must hold in order for the singular solitons to exist. Again the singular soliton profile for $h(x-v t)$ can be obtained from (60).

\section{CONCLUSIONS}

IJEF shows that soliton solutions and triangular periodic solutions can be established as the limits of Jacobi doubly periodic wave solutions. When $m \longrightarrow 1$, the Jacobi functions degenerate to the hyperbolic functions and given the solutions by the extended hyperbolic functions methods. When $m \longrightarrow 0$, the Jacobi functions degenerate to the triangular functions and given the solutions by extended triangular functions methods. Additionally, the direct traveling wave hypothesis also lead to solitray waves and singular soliton solutions, for the non-dissipative Broer-Kaup equation. In this case there are several constraint conditions that fell out in order for 
the solitons to exist.

These results will be discussed further in future where several other aspects will be addressed. These are the conservation laws [34], perturbed Broer-Kaup system including the stochastic perturbation. Additionally, full numerical simulations will be carried out and these results will all be declared in future publications.

\section{Acknowledgments}

This work was funded by the Deanship of Scientific Research (DSR), King Abdulaziz University under grant number (14-130/1433 HiCi). The authors therefore thankfully acknowledge technical and financial support of KAU.

\section{References}

[1] D.J. Kaup, Progress of Theoretical Physics, 54, 396-408 (1975).

[2] M. Wang, J. Zhang and X. Li, Applied Mathematics and Computation, 206, 321-326 (2008).

[3] S. Guo, Y. Zhou and C. Zhao, Applied Mathematics and Computation, 216, 1965-1971 (2010).

[4] Z. Zheng and W.-R. Shan, Applied Mathematics and Computation, 215, 2390-2396 (2009).

[5] M. Rafei and H. Daniali, Computers and Mathematics with Applications, 54, 1079-1085 (2007).

[6] Zh. Yan and H. Zhang, Physics Letters A, 285, 355-362 (2001).

[7] Z. Ping, Applied Mathematics and Computation, 217, 16881696 (2010).

[8] G.B. Whitham, Proc. Roy. Soc. London Ser. A, 299, 6-25 (1967).

[9] L. J. F. Broer, Appl. Sci. Res., 31, 377-395 (1975).

[10] B. A. Kupershmidt, Commun. Math. Phys., 99, 51-53 (1985).

[11] E. Yomba, Phys. Lett. A, 336, 463-476 (2005).

[12] A.H. Bhrawy and M. A. Abdelkawy, Indian Journal of Physics (2013), DOI:10.1007/s 12648-013-0260-1.

[13] M.L. Wang, Phys. Lett. A, 199, 169-172 (1995).

[14] Z.Y. Yan and H.Q. Zhang, Phys. Lett. A, 252, 291-296 (1999).

[15] C.L. Chen and S.Y. Lou, Chaos Solitons Fract., 16, 27-35 (2003).

[16] X.D. Zheng, Y. Chen and H.Q. Zhang, Phys. Lett. A, 311, 145-157 (2003)

[17] X. Zeng and D.-S. Wangb, Applied Mathematics and Computation, 212, 296-304 (2009).

[18] S. Ghosh and S. Nandy, Nuclear Physics B, 561, 451-466 (1999).

[19] R.A. Baldock, B.A. Robson and R.F. Barrett, Nuclear Physics A, 366, 270-280 (1981).

[20] A. M. Wazwaz, Applied Mathematics and Computation, 200, 160-166 (2008).

[21] J. F. Zhang, Physics Letters A, 313, 401-407 (2003).
[22] M. Yaghobi Moghaddam, A. Asgari and H. Yazdani, Applied Mathematics and Computation, 210, 422-435 (2009).

[23] S. Tang, C. Li and K. Zhang, Communications in Nonlinear Science and Numerical Simulation, 15, 3358-3366 (2010).

[24] A. H. Bhrawy, M. A. Abdelkawy, S. Kumar, S. Johnson, A. Biswas, Indian Journal of Physics, 87, 455-463 (2013)

[25] V. N. Serkin, V.M. Chapela, J. Percino, T.L. Belyaeva, Nonlinear tunneling of temporal and spatial optical solitons through organic thin films and polymeric waveguides, Optics Communications, 192, 237-244 (2001).

[26] A. H. Khater, D. K. Callebaut and M. A. Abdelkawy, Phys. of plasmas, 17, 122902 (2010).

[27] A.H. Khater, D.K. Callebaut, A.H. Bhrawy and M.A. Abdelkawy, Journal of Computational and Applied Mathematics, 242, 28-40 (2013).

[28] A. H. Bhrawy and M. A. Abdelkawy, Central European Journal of Physics, (2013), doi:10.2478/s11534-013-0197-1.

[29] A. H. Bhrawy, M. A. Abdelkawy and A. Biswas, Communications in Nonlinear Science and Numerical Simulation, 18, 915-925 (2013).

[30] A. H. Bhrawy, A. Yildirim, M. M. Tharwat and M. A. Abdelkawy, Indian Journal of Physics, 86, 1107-1113 (2012).

[31] A. V. Porubov and D. F. Parker, Wave Motion, 29, 97-108 (1999).

[32] E. Fan, J. Phys. A: Math. Gen., 35, 6853-6872 (2002).

[33] M. Song, Z. Liu, E. Zerrad and A. Biswas, Applied Mathematics and Information Sciences, 7, 1333-1340 (2013).

[34] Nikolai A. Kudryashov, Dmitry I. Sinelshchikov, Maria V. Demina, Exact solutions of the generalized Bretherton equation, Physics Letters A, 375, 1074-1079 (2011).

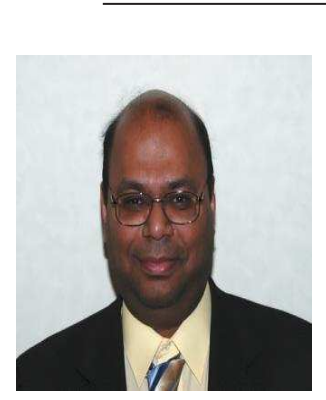

Anjan Biswas earned his B.Sc (hons) degree in Mathematics from St. Xavier's College in Calcutta, India. Subsequently, he earned his M.Sc and M.Phil degrees from the University of Calcutta. After that he further earned his MA and Ph.D. degrees from the University of New Mexico in Albuquerque, NM, USA. Currently, he is an Associate Professor in the Department of Mathematical Sciences at Delaware State University in Dover, DE. His research interest is on Theory of Solitons applicable to Physics, Engineering and Biosciences. 